\title{
Ultralow Voltage Manipulation of Ferromagnetism
}

\author{
Bhagwati Prasad,* Yen-Lin Huang, Rajesh V. Chopdekar, Zuhuang Chen, James Steffes, \\ Sujit Das, Qian Li, Mengmeng Yang, Chia-Ching Lin, Tanay Gosavi, Dmitri E. Nikonov, \\ Zi Qiang Qiu, Lane W. Martin, Bryan D Huey, lan Young, Jorge Íñiguez, \\ Sasikanth Manipatruni, and Ramamoorthy Ramesh*
}

Spintronic elements based on spin transfer torque have emerged with potential for on-chip memory, but they suffer from large energy dissipation due to the large current densities required. In contrast, an electric-field-driven magnetoelectric storage element can operate with capacitive displacement charge and potentially reach $1-10 \mu \mathrm{J} \mathrm{cm}{ }^{-2}$ switching operation. Here, magneto-electric switching of a magnetoresistive element is shown, operating at or below $200 \mathrm{mV}$, with a pathway to get down to $100 \mathrm{mV}$. A combination of phase detuning is utilized via isovalent La substitution and thickness scaling in multiferroic $\mathrm{BiFeO}_{3}$ to scale the switching energy density to $\approx 10 \mu \mathrm{J} \mathrm{cm}{ }^{-2}$. This work provides a template to achieve attojoule-class nonvolatile memories.

applications are beginning to highlight the limitations imposed by the "memory wall" on computing (in terms of the number of operations per Joule). ${ }^{[8]}$ At the same time, the techno-economic innovation driver behind semiconductor electronics, namely Moore's law, is slowing down mainly as a consequence of the critical dimensions reaching below $10 \mathrm{~nm}$ with a concomitant increase in power consumption. ${ }^{[9]}$ These coupled pressures have set the stage for an intense worldwide search for the next logic/memory device that can replace Si-CMOS. In par-

New approaches for logic and memory functions are lately of tremendous scientific and technological interest as a consequence of substantially increasing global demand projections, corresponding concerns for energy consumption, and the commercial necessity for future dimensional scalability. ${ }^{[1-3]}$ This is driven by the exponential increase in the deployment of microelectronic components embedded into the "Internet of Things", computational and memory intensive emerging applications such as machine learning, and the increasing ubiquity of ultrahigh-resolution video. ${ }^{[4-7]}$ These emerging allel, the solid-state memory market has been booming in recent years ${ }^{[8,10,11]}$ heightening the potential value of a universal memory device which is yet to be implemented.

Both of these functionalities are provided by a new, magnetoelectric spin-orbit (MESO) coupled device, ${ }^{[12]}$ which implements a new generation of quantum materials, including ferromagnets, ferroelectrics, magnetoelectrics, and spin-orbit coupled materials. MESO is based on electric field manipulation of the magnetic states of a spintronic device, by exploiting the magnetoelectric coupling in multiferroic materials, thereby achieving logic and
Dr. B. Prasad, ${ }^{[+]}$Dr. Y.-L. Huang, Dr. Z. Chen, Dr. S. Das,

Prof. L. W. Martin, Prof. R. Ramesh

Department of Materials Science and Engineering

University of California

Berkeley, CA 94720, USA

E-mail: bhagwati.prasad@wdc.com; rramesh@berkeley.edu

Dr. Y.-L. Huang, Prof. L. W. Martin

Materials Sciences Division

Lawrence Berkeley Laboratory

Berkeley, CA 94720, USA

Dr. R. V. Chopdekar

Advanced Light Source

Lawrence Berkeley National Laboratory

Berkeley, CA 94720, USA

Dr. J. Steffes, Prof. B. D. Huey

Institute of Materials Science

University of Connecticut

Storrs, CT 06269, USA

The ORCID identification number(s) for the author(s) of this article can be found under https://doi.org/10.1002/adma.202001943.

${ }^{[+]}$Present address: Western Digital Research Center, Western Digital

Corporation, San Jose, California 95119, USA

${ }^{[++}$Present address: Kepler Computing, Hillsboro, OR 97124, USA

\author{
Dr. Q. Li, Dr. M. Yang, Prof. Z. Q. Qiu, Prof. R. Ramesh \\ Department of Physics \\ University of California \\ Berkeley, CA 94720, USA \\ Dr. C.-C. Lin, Dr. T. Gosavi, Dr. D. E. Nikonov, Dr. I. Young, \\ Dr. S. Manipatruni ${ }^{[++}$ \\ Exploratory Integrated Circuits \\ Components Research \\ Intel Corp. \\ Hillsboro, OR 97124, USA \\ Prof. J. Íñiguez \\ Materials Research and Technology Department \\ Luxembourg Institute of Science and Technology (LIST) \\ Avenue des Hauts-Fourneaux 5, Esch-sur-Alzette L-4362, Luxemburg \\ Prof. J. Íñiguez \\ Physics and Materials Science Research Unit \\ University of Luxembourg \\ 41 Rue du Brill, Belvaux L-4422, Luxembourg \\ Dr. S. Manipatruni \\ Kepler Computing \\ Hillsboro, OR 97124, USA
}

DOI: 10.1002/adma.202001943 
memory functionalities in the attojoule energy class. ${ }^{[9]}$ The desirable magnetoelectric mechanism is driven by switching of the ferroelectric component, since the energy density for polarization reversal $\left(E / A=2 \times P_{\mathrm{S}} \times V_{\mathrm{FE}}\right.$, where $P_{\mathrm{S}}$ is the saturation polarization per unit area, $V_{\mathrm{FE}}$ is the ferroelectric switching voltage) can reasonably be scaled to $1 \mu \mathrm{J} \mathrm{cm}^{-2}$. The multiferroic nature of the switching medium enables nonvolatility (relying on the FE polarization $P$ and the antiferromagnetic order $L$ ), directional coupling, and signal retention. ${ }^{[2,13]}$ However, achieving 1 attojoule requires significant innovations in materials physics; principally a reduction of the switched polarization down to less than $10 \mu \mathrm{C} \mathrm{cm}-2$ with a concomittant switching voltage below $100 \mathrm{mV}$ and thus this represents a grand challenge for the ferroic community.

With this as the background, we set out to explore the limits of ferroelectric and magnetoelectric coupling/switching using the $\mathrm{BiFeO}_{3}$ model system. Here we demonstrate magnetoelectric switching of a magnetic element in order to demonstrate the proof of concept of electric field manipulation of the magnetic coupling, operating at or below $200 \mathrm{mV}$. We obtain this by designing lower-voltage ferroelectric switching in $\mathrm{Bi}_{1-x} \mathrm{La}_{x} \mathrm{FeO}_{3}$ and leveraging innate thickness scaling effects and high-quality interfaces with conductive oxide electrodes to overcome the asymmetry intrinsic to a magnetoelectric with a metallic, magnetoresistance readout to demonstrate the viability of this approach.

The magnetoelectric coupling test structures were prepared using a model $\mathrm{Co}_{0.9} \mathrm{Fe}_{0.1}-\mathrm{Cu}-\mathrm{Co}_{0.9} \mathrm{Fe}_{0.1}$ based magnetic spinvalve structure integrated onto the $\mathrm{Bi}_{0.85} \mathrm{La}_{0.15} \mathrm{FeO}_{3}$ (BLFO) surface in ultrahigh vacuum (Figure 1a, also Experimental Section). We preface our discussion by noting that the intention behind this experiment was not to demonstrate a specific technological manifestation (i.e., a spin valve operation) but instead to use it to probe the interfacial coupling. In brief, Pt $(2 \mathrm{~nm}) / \mathrm{Co}_{0.9} \mathrm{Fe}_{0.1}(2.5 \mathrm{~nm}) / \mathrm{Cu} \quad(5 \mathrm{~nm}) / \mathrm{Co}_{0.9} \mathrm{Fe}_{0.1}$
(2.5 nm) metallic multilayer thin films are deposited on $\mathrm{BLFO} / \mathrm{La}_{0.7} \mathrm{Sr}_{0.3} \mathrm{MnO}_{3} / \mathrm{SrRuO}_{3}$ trilayer oxide films. Notably, a few nanometers $\left(3-5 \mathrm{~nm}\right.$ ) of $\mathrm{La}_{0.7} \mathrm{Sr}_{0.3} \mathrm{MnO}_{3}$ (LSMO) inserted between the $\mathrm{Bi}_{1-x} \mathrm{La}_{x} \mathrm{FeO}_{3}$ and $\mathrm{SrRuO}_{3}$ (SRO) is found to dramatically reduce the asymmetric nature of the ferroelectric state that otherwise arises due to the differences in work function arising from the $\mathrm{SrRuO}_{3}$ bottom and $\mathrm{Co}_{0.9} \mathrm{Fe}_{0.1}$ top electrodes (see Figure S1, Supporting Information). Conventional photolithography is employed to fabricate several spin-valve devices with sizes ranging from $\left(2 \times 15 \mu \mathrm{m}^{2}\right)$ to $\left(10 \times 50 \mu \mathrm{m}^{2}\right)$ (see Experimental Section). Magnetization hysteresis loops of the $\mathrm{Co}_{0.9} \mathrm{Fe}_{0.1}(2.5 \mathrm{~nm}) / \mathrm{Cu}(5 \mathrm{~nm}) / \mathrm{Co}_{0.9} \mathrm{Fe}_{0.1}(2.5 \mathrm{~nm})$ spin valve (Figure 1b), illustrate that the bottom $\mathrm{Co}_{0.9} \mathrm{Fe}_{0.1}$ layer couples magnetically to the BLFO with an exchange enhancement of the coercive field by $\approx 100$ Oe, while the top, free $\mathrm{Co}_{0.9} \mathrm{Fe}_{0.1}$ layer exhibits a lower coercive field, thus fulfilling the requirement of achieving the two opposing magnetic states (parallel and antiparallel) that can be probed in a magnetotransport measurement. The magnitude of the exchange interaction at the interface decreases slightly with additional lanthanum substitution (see Figure S2, Supporting Information); this is an indication of subtle changes in the strength of the DzyaloshinskiiMoriya interaction (DMI) in the $\mathrm{Bi}_{1-x} \mathrm{La}_{x} \mathrm{FeO}_{3}$ layer, possibly because of different degree of lattice distortion and spin-orbital coupling induced lanthanum substitution. More importantly, such a strong coupling at the $\mathrm{Bi}_{1-x} \mathrm{La}_{x} \mathrm{FeO}_{3} / \mathrm{Co}_{0.9} \mathrm{Fe}_{0.1}$ interface directly points to the existence of an adequately robust canted moment and therefore DMI. X-ray magnetic linear dichroism spectra confirm that the BLFO behaves similarly to $\mathrm{BiFeO}_{3}$ in terms of antiferromagnetism (see Figure S3, Supporting Information). A representative in-plane magnetoresistance plot of a spin-valve device integrated with multiferroic BLFO is shown (Figure $1 c$ ). We typically observe $\approx 1 \%$ giant magnetoresistance
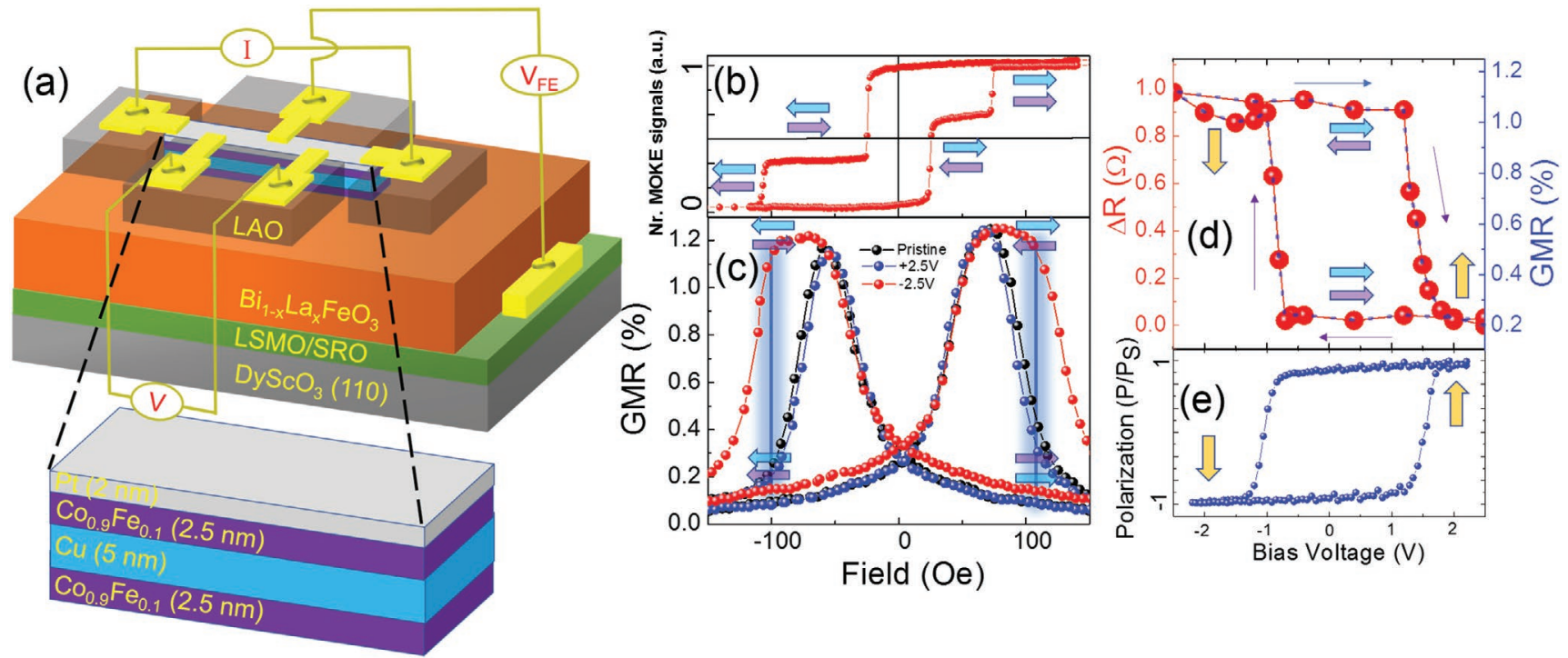

Figure 1. Magnetoelectric coupling in La doped BFO. a) Schematic of the testing protocol of magnetoelectric coupling in $\mathrm{Bi}_{1-x} \mathrm{La}_{x} \mathrm{FeO}_{3}$ system, established by integrating the $\mathrm{Co}_{0.9} \mathrm{Fe}_{0.1}(2.5 \mathrm{~nm}) / \mathrm{Cu}(5 \mathrm{~nm}) / \mathrm{Co}_{0.9} \mathrm{Fe}_{0.1}(2.5 \mathrm{~nm})$ spin valve on multiferroic $\mathrm{Bi}_{0.85} \mathrm{La}_{0.15} \mathrm{FeO}_{3}(\mathrm{BLFO})$ with $\mathrm{La}_{0.7} \mathrm{Sr}_{0.3} \mathrm{MnO}_{3} / \mathrm{SrRuO}_{3}$ as the bottom electrode on $\mathrm{DySCO}_{3}(110)$ substrates. b) Magnetization hysteresis loop of a $\mathrm{Co}_{0.9} \mathrm{Fe}_{0.1}(2.5 \mathrm{~nm}) / \mathrm{Cu}(5 \mathrm{~nm}) / \mathrm{Co}_{0.9} \mathrm{Fe}_{0.1}(2.5 \mathrm{~nm})$ trilayer structure, where the bottom $\mathrm{Co}_{0.9} \mathrm{Fe}_{0.1}$ layer strongly magnetically coupled with the canted moments of $\mathrm{BLFO}$ film ( $\left.\approx 50 \mathrm{~nm}\right)$. c) Modulation of the $\mathrm{GMR}$ response of a patterned spin valve device upon the application of $\pm 2.5 \mathrm{~V}$ across the BLFO film along with the GMR response for pristine case. $d$ ) Modulation of resistance state (parallel/antiparallel) of the spin valve structure with applied voltage across BLFO and e) corresponding ferroelectric $P-V$ loop. 
(GMR) ratio in these devices, which is sufficiently high to detect the magnetoelectric coupling in BLFO. Similar to a conventional GMR device, the switching fields corresponding to the two resistance states (low and high) for the pristine case (before electric bias) match well with the coercive fields of the two magnetic layers. Upon application of $\pm 2.5 \mathrm{~V}$ the magnetotransport in the GMR structure can be systematically modulated as shown in Figure 1c. Specifically, this shows that the magnetic coercive field of the bottom $\mathrm{Co}_{0.9} \mathrm{Fe}_{0.1}$ layer in the GMR stack is being altered by the application of the electric field. For example, at a fixed magnetic field of -100 Oe, an applied voltage of $-2.5 \mathrm{~V}$ leads to a large GMR signal $(\approx 1.2 \%)$ corresponding to the high-resistance state (i.e., the moments in the top and bottom $\mathrm{Co}_{0.9} \mathrm{Fe}_{0.1}$ layers are antiparallel). In contrast, an applied voltage of $+2.5 \mathrm{~V}$ leads to a low GMR resistance state (i.e., moments in the top and bottom $\mathrm{Co}_{0.9} \mathrm{Fe}_{0.1}$ layers are aligned parallel). These changes in the resistance of the GMR device are captured (Figure 1d) as a voltage-dependent hysteresis that closely follows the ferroelectric switching of the BLFO layer, demonstrated by the ferroelectric polarization loop (Figure 1e). Such a one-to-one correlation between ferroelectric and magnetoelectric switching has been observed in all devices even with varying BLFO thicknesses (e.g., see Figure S4, Supporting Information, for $35 \mathrm{~nm}$ BLFO).

The switching voltage of the GMR response can be progressively reduced from $\approx 1 \mathrm{~V}$ to $500 \mathrm{mV}$ by a reduction of the film thickness down to $20 \mathrm{~nm}$ (Figure 2a). Such a switching behavior was very reproducible and the resistance transition became sharper as the device dimension was reduced (see Figure S5, Supporting Information). To ensure that such an electric-field control of the magnetization direction in the bottom $\mathrm{Co}_{0.9} \mathrm{Fe}_{0.1}$ layer is not driven by the magnetic field that is used to carry out the
GMR measurements, we carried out these measurements both in a magnetic field of 100 Oe as well as in the remanent state (i.e., zero magnetic field). As shown (Figure 2b), this confirms that the magnetic field applied for the GMR measurements does not influence the magnetoelectric coupling since both the zero field and 100 Oe field hysteresis loops are essentially identical. We further corroborate the low-voltage magnetoelectric switching in multiferroic $\mathrm{Bi}_{1-x} \mathrm{La}_{x} \mathrm{FeO}_{3}$ by X-ray magnetic circular-dichroismbased photoemission electron microscopy (XMCD-PEEM) imaging at the Co $L_{3}$ edge via studies of patterned Pt $(2 \mathrm{~nm}) /$ $\mathrm{Co}_{0.9} \mathrm{Fe}_{0.1}(2.5 \mathrm{~nm})$ structures on $20 \mathrm{~nm} \mathrm{Bi}{ }_{0.85} \mathrm{La}_{0.15} \mathrm{FeO}_{3}$. As shown in the XMCD-PEEM images (inset, Figure 2c), after the application of -500 and $+500 \mathrm{mV}$, there is a contrast reversal which signifies a corresponding reversal of the in-plane magnetization.

Further reductions of the switching voltage were explored by reducing the BLFO thickness progressively down from 20 to $5 \mathrm{~nm}$. As expected, at such thicknesses, leakage plays an increasingly dominant role in determining transport across the layer. Therefore, we studied the magnetoelectric coupling between a $10 \mathrm{~nm}$ BLFO layer and the ferromagnetic $\mathrm{Co}_{0.9} \mathrm{Fe}_{0.1}$ (2.5 $\mathrm{nm})$ using a combination of piezoforce microscopy to probe the polar order (Figure S6, Supporting Information) and XMCD-PEEM based imaging of the ferromagnetic state in the layer in contact with the BLFO. The results of this experiment are captured in Figure 2d, which shows that the magnetic state can indeed be switched with an electric voltage of $\pm 200 \mathrm{mV}$, cor-

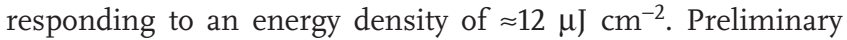
magnetotransport results (Figure S6, Supporting Information) support this conclusion.

Our pathway to design the energy landscape of $\mathrm{BiFeO}_{3}$ uses first-principles calculations to comprehend the influence of local electronic structure on the structural stability, polar
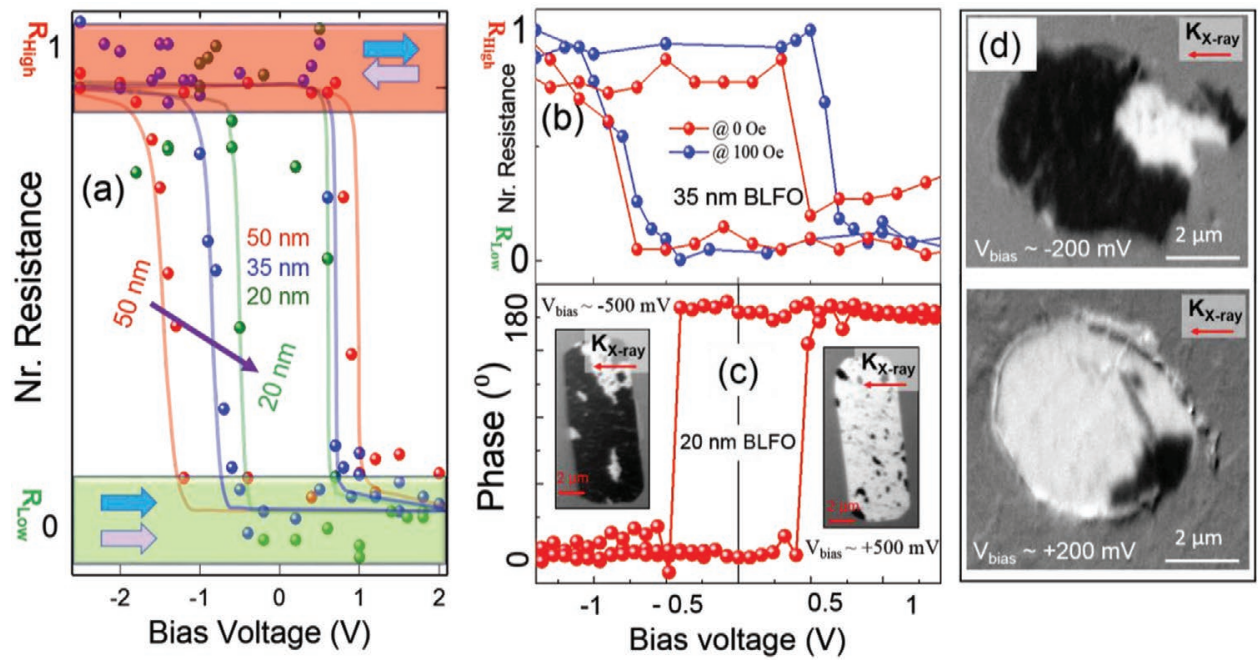

Figure 2. Scaling of switching energy. a) The modulation of the resistance state (normalized resistance) of a spin valve device structured on BLFO films, upon the application of varying bias voltage across BLFO layer with different film thicknesses. A background magnetic field of 100 Oe is applied to set the state. Solid lines are a guide to the eye. b) The trend of resistance modulation of a spin valve device, integrated with $\approx 35 \mathrm{~nm} B L F O$ film, with a 100 Oe of background field is the same as that without any magnetic field, thereby emphasizing that the background magnetic field does not play any role in electric field modulation of the resistance state of the spin valve. c) Piezoelectric loop of a $20 \mathrm{~nm}$ BLFO film, showing the ferroelectric switching voltage $\approx \pm 500 \mathrm{mV}$. The XMCD-PEEM images (inset) of the $\mathrm{Pt} / \mathrm{Co}_{0.9} \mathrm{Fe}_{0.1}(2.5 \mathrm{~nm})$ strips, obtained with a presetting magnetic field pulse of $\approx 100$ Oe, reveal the $180^{\circ}$ reversal of magnetization upon the application of $\pm 500 \mathrm{mV}$ across the BLFO. d) XMCD-PEEM images of Pt/Co.9. $\mathrm{Fe}_{0.1}(2.5 \mathrm{~nm})$ disks coupled to a $10 \mathrm{~nm}$ BLFO layer showing a further reduction of the switching voltage down to $\pm 200 \mathrm{mV}$. $K_{X \text {-ray }}$ is the in-plane component of the incident $X$-ray beam from right to the left. 

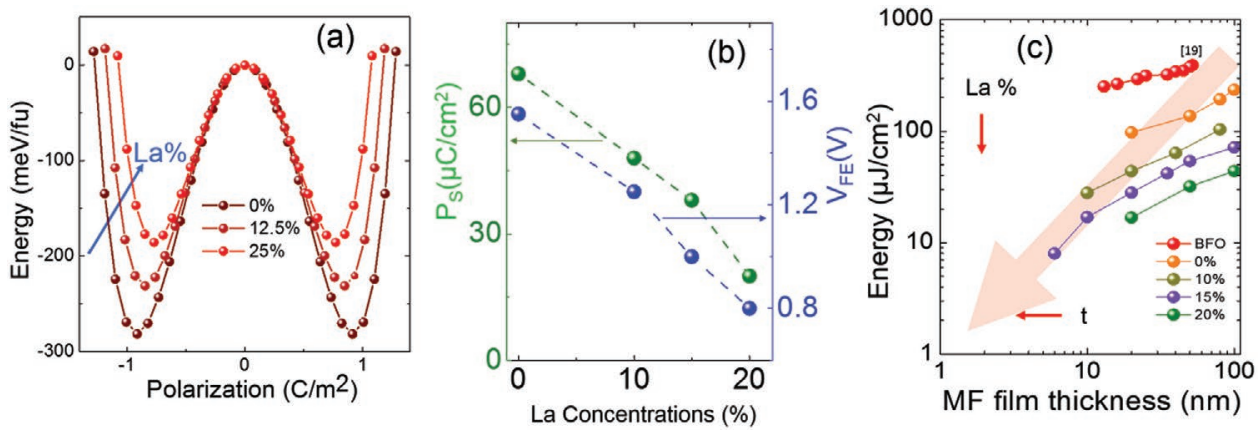

Figure 3. Tuning the Landau energy landscape of BFO with La substitution at Bi site. a) Theoretical modeling data, obtained from first-principles calculations, showing the evolution of the free energy of BFO with varying La doping. b) Variation of saturation polarization $\left(P_{\mathrm{S}}\right)$ and ferroelectric switching voltage $\left(V_{\mathrm{FE}}\right)$ with La concentration of $100 \mathrm{~nm}$ thin films. c) Scaling of energy density with varying BLFO film thickness $(t)$ and La doping.

order, antiferromagnetism, and the magnetoelectric coupling. Replacing bismuth with isovalent lanthanum reduces the polarization by $\approx 40 \%[14,15]$ (Figure 3a), while the switching barrier height decreases by $\approx 50 \%$ compared to pure $\mathrm{BiFeO}_{3}$. Our theoretical calculations also revealed that beyond $\approx 30 \%$ of lanthanum substitution there is a finite probability for the occurrence of other crystallographic phases. ${ }^{[16]}$ The evolution of the out-of-plane lattice parameter with increasing lanthanum substitution, derived from X-ray diffraction studies (see Figure S7a, Supporting Information), clearly shows that the structure of the $\mathrm{Bi}_{1-x} \mathrm{La}_{x} \mathrm{FeO}_{3}$ unit cell systematically changes with lanthanum concentration. Specifically, the out-of-plane lattice parameter progressively diminishes with increasing lanthanum content. These structural observations correspond to an equivalent change in the spontaneous polarization (see Figure S7b, Supporting Information), ${ }^{[15-17]}$ which is indeed observed in $100 \mathrm{~nm}$ thick films used as a reference (see Figure 3b). The spontaneous polarization decreases by $\approx 60 \%$ while the coercive field decreases by $\approx 50 \%$, which is qualitatively consistent with the theoretical predictions. We also observed a direct, one-to-one correlation between the ferroelectric and antiferromagnetic domains of BLFO before and after electric poling as in $\mathrm{BiFeO}_{3},{ }^{[18]}$ clearly showing that the antiferromagnetic (and thus the canted moment) axis rotates along with the ferroelectric polarization upon the application of an electric field (see Figure S8, Supporting Information). This also reflects on the spin-valve transport data (discussed in Figure 1), since the projection of the canted moment in the plane will directly change the strength of the exchange coupling between the bottom $\mathrm{Co}_{0.9} \mathrm{Fe}_{0.1}$ layer and $\mathrm{Bi}_{1-x} \mathrm{La}_{x} \mathrm{FeO}_{3}$ layer.

In summary, we have demonstrated the cooperative effects of both the La substitution and thickness to enable a reduction of both the spontaneous polarization and the height of the energy barrier in the double well structure (Figure 3a), thus enabling a reduction of the energy density down to below $10 \mu \mathrm{J} \mathrm{cm}^{-2}$ which is summarized in Figure 3c (data for $\mathrm{BiFeO}_{3}$ from ref. [19] also included for comparison). We note that it already puts this multiferroic magnetoelectric pathway at a lower energy level compared to known memory technologies. ${ }^{[11,19-22]}$ Reductions of the energy density down to $1 \mu \mathrm{J} \mathrm{cm}^{-2}$ will require further tuning of the switched polarization of the $\mathrm{BiFeO}_{3}$ down to $\approx 10 \mu \mathrm{C} \mathrm{cm}{ }^{-2}$ and the coercive voltage $(<100 \mathrm{mV})$. As shown in Figure $2 \mathrm{~d}$ and Figure S6, Supporting Information, we have existential proof that the switching voltage can indeed be reduced to $\approx 200 \mathrm{mV}$ for a $10 \mathrm{~nm}$ BLFO film and further reduction of switching voltage down to $\approx 100 \mathrm{mV}$ can be achieved with $\approx 5 \mathrm{~nm}$ thick magnetoelectric layers (see Figure S9a-c, Supporting Information). The magnetic exchange coupling between the BLFO and thin $\mathrm{Co}_{0.9} \mathrm{Fe}_{0.1}$ layer is still significant enough for BLFO thickness down to $5 \mathrm{~nm}$ (see Figure S9d, Supporting Information). We note that there have been very little detailed studies of the switching dynamics of the polar state in ferroelectrics (and by default multiferroics) at such thicknesses, although there have been numerous studies of the stability of the polar ground state. ${ }^{[23-25]}$ A lot more detailed measurements of the switchability of the polar state and therefore the magnetoelectric coupling for multiferroic film thicknesses below $10 \mathrm{~nm}$ would be extremely useful.

\section{Experimental Section}

Film Growth: The oxide heterostructures BLFO/LSMO/SRO were grown on single-crystalline (110) $\mathrm{DySCO}_{3}$ by pulsed laser deposition at $690-710{ }^{\circ} \mathrm{C}$ with focused laser fluence $\approx 1.2 \mathrm{~J} \mathrm{~cm}^{-2}$ under $100-160 \mathrm{mTorr}$ oxygen pressure and cooled down to room temperature under 500 Torr oxygen pressure. After the cooling process, the oxide heterostructures were immediately transferred to high vacuum magnetron sputtering chamber with a base pressure $\approx 1 \times 10^{-7}$ Torr. The spin valve structures used in this article were fabricated with $\mathrm{Pt}(2.5 \mathrm{~nm}) / \mathrm{Co}_{0.9} \mathrm{Fe}_{0.1}(2.5 \mathrm{~nm}) /$ $\mathrm{Cu}(3-5 \mathrm{~nm}) / \mathrm{Co}_{0.9} \mathrm{Fe}_{0.1}$, deposited by $\mathrm{DC}$ magnetron sputtering with argon pressure ranging from $2 \times 10^{-3}$ to $7 \times 10^{-3}$ Torr under a static magnetic field of 200 Oe along the crystallographic [001]。 to establish the magnetic easy axis. A $2 \mathrm{~nm}$ Pt layer was deposited on top of the spin valve as a capping layer to protect the top $\mathrm{Co}_{0.9} \mathrm{Fe}_{0.1}$ layer from oxidation, whereas the bottom $\mathrm{SrRuO}_{3}$ layer serves as a bottom electrode for ferroelectric switching.

Fabrication of Spin Valve Devices and Magnetoelectric Coupling Measurements: Conventional photolithography and Ar-ion milling were employed to pattern the $\mathrm{Co}_{0.9} \mathrm{Fe}_{0.1}(2.5 \mathrm{~nm}) / \mathrm{Cu}(5 \mathrm{~nm}) / \mathrm{Co}_{0.9} \mathrm{Fe}_{0.1}(2.5 \mathrm{~nm})$ spin valves devices of $15 \times 5 \mu \mathrm{m}^{2}$. Subsequently, a $200 \mathrm{~nm}$ thick insulating amorphous $\mathrm{LaAlO}_{3}$ (LAO) film was selectively deposited on BLFO surface by pulsed laser deposition to isolate the connecting lines and contact pads from BLFO. DC magnetron sputtering was used to deposit $230 \mathrm{~nm}$ thick Au film for electrical contacts. The GMR responses of spin valve devices were measured in current-in-plane configuration at constant current with varying magnetic field. The magnetoelectric coupling measurements were conducted with the application of electric field pulse (10-100 $\mu \mathrm{s})$ across BLFO films. For nanoscale device fabrication, e-beam lithography and Ar ion-milling processes were used 
to pattern $\mathrm{Co}_{0.9} \mathrm{Fe}_{0.1}(2.5 \mathrm{~nm}) / \mathrm{Cu}(5 \mathrm{~nm}) / \mathrm{Co}_{0.9} \mathrm{Fe}_{0.1}(2.5 \mathrm{~nm})$ spin valves. With a second e-beam lithography step, $20 \mathrm{~nm}$ sputtered $\mathrm{SiO}_{2}$ layer was patterned to avoid the top electrode shorting to the bottom $\mathrm{SrRuO}_{3}$ electrode. Finally, the third e-beam lithography step, was used to pattern $5 \mathrm{~nm} \mathrm{Ti} / 50 \mathrm{~nm} \mathrm{Au}$ as a top electrode.

Soft X-ray Microscopy and Spectroscopy: X-ray imaging with variable circular and linear polarization at the $\mathrm{Co}$ and $\mathrm{Fe} L$ edges was performed at the PEEM3 end station of BL11.0.1 at the Advanced Light Source, Lawrence Berkeley National Lab. The sample was held at an angle of $60^{\circ}$ with respect to the surface normal, and was mounted on an azimuthal rotation stage. The in-plane azimuthal angle of zero degrees was with $X$-rays incident along the in-plane [-100] direction. The sample was held at a voltage of $-18 \mathrm{kV}$ to accelerate any photoemitted and secondary electrons, proportional to the local X-ray absorption coefficient, through a series of electrostatic lenses and incident onto a CCD detector with a phosphor-coated fiber plate serving as an amplifier. Ferromagnetic domains in the CoFe layer were measured by taking the difference between $1 \mathrm{~s}$ exposures taken with right and left circularly polarized $X$-rays at the $\mathrm{Co}_{3}$ edge to emphasize the XMCD signal and to minimize topographic and work function contrast. The XMCD contrast was proportional to angle between the ferromagnetic domain orientation and the $\mathrm{X}$-ray incidence angle. To probe antiferromagnetic orientation projections along the $X$-ray linear polarization axis, linear dichroism images at the $\mathrm{Fe} \mathrm{L}_{2} \mathrm{~A}$ and $\mathrm{B}$ edges of 720.6 and $722 \mathrm{eV}$ were taken at five linear polarization axis angles between s- and p-polarization. For each polarization value, a pre-edge image at $718 \mathrm{eV}$ was taken to normalize the on-edge images. A series of ten images with $2 \mathrm{~s}$ acquisition time each were taken at each energy and polarization angle, and the entire polarization angle-energy sequence was repeated four times to improve statistics. For XMCD-PEEM imaging of $\mathrm{Pt} / \mathrm{Co}_{0.9} \mathrm{Fe}_{0.1} /$ BLFO samples, the magnetization of the $\mathrm{Co}_{0.9} \mathrm{Fe}_{0.1}$ layer was first saturated with $\approx \pm 300$ Oe of magnetic field and then an electric pulse of $\approx \pm 500 \mathrm{mV} / \pm 200 \mathrm{mV}$ was applied, and subsequently the XMCD-PEEM image was taken after setting a magnetic field pulse of $\approx 100$ Oe. For XMLD spectroscopy measurements, Fe $L$ edge spectra were taken in a normal incidence geometry at BL4.0.2 at the Advanced Light Source, Lawrence Berkeley National Lab.

First-Principles Calculations: For the calculations density functional theory was used as implemented in the Vienna Ab inititio Simulation Package (VASP) software. ${ }^{[26]}$ For the density functional, the generalized gradient approximation introduced in ref. [27] was used, which has been shown to be the option of choice when discussing the relative stability of rhombohedral and orthorhombic phases in $\mathrm{BiFeO}_{3}$-related compounds. ${ }^{[28]}$ Also, as regularly done, a "Hubbard U" was used for correction for a better treatment of iron's 3d electrons. ${ }^{[29]}$ Electronic wave functions were represented in a plane-wave basis cut off at $500 \mathrm{eV}$. The ionic cores were treated within the so-called projector-augmented wave approximation, ${ }^{[30]}$ considering the following electrons explicitly in the simulations: Fe's $3 p, 3 d$, and 4s; Bi's $6 s$ and $6 p$; La's $5 s, 5 p, 5 d$, and $6 \mathrm{~s}$; and $\mathrm{O}$ 's $2 \mathrm{~s}$ and $2 \mathrm{p}$. Most of our calculations involve a 40 -atom supercell that can be seen as a $2 \times 2 \times 2$ multiple of the elemental fiveatom perovskite unit. Integrals in the corresponding Brillouin zone were computed using a $2 \times 2 \times 2 \mathrm{k}$-point grid. Structural relaxations were run until the residual forces on the atoms fall below $0.01 \mathrm{eV} \mathrm{A}^{-1}$. These calculation conditions were checked for sufficient convergence.

Different $\mathrm{Bi}-\mathrm{La}$ compositions were considered by substituting part of the eight $\mathrm{Bi}$ atoms in our supercell with $\mathrm{La}$, which allows to study composition steps of $12.5 \%$. For compositions $0 \%$ and $12.5 \%$, only one symmetry-inequivalent $\mathrm{Bi}-\mathrm{La}$ arrangement was allowed in our supercell, while many were possible for compositions $25 \%$ and $37.5 \%$; in the latter cases, two atomic configurations were considered, and checked that the qualitative results do not depend strongly on the $\mathrm{Bi}-\mathrm{La}$ cationic order.

To investigate the preference for one-step versus multi-step ferroelectric switching, the activation energy associated to the full and minimal polarization rotations were compared, which in the case of $\mathrm{BiFeO}_{3}$ correspond to the highest and lowest barriers, respectively. The material was always considered to be in a mono-domain configuration, and the potential energy landscape investigated corresponds to the limit of very low temperature (strictly speaking, $0 \mathrm{~K}$ ); note that both approximations can be expected to result in much exaggerated switching barriers and coercive fields as compared to the experimental situation at room temperature. The corresponding switching paths were obtained from direct interpolation between well-defined start, medium, and end points, which drastically simplifies the calculations.

\section{Supporting Information}

Supporting Information is available from the Wiley Online Library or from the author.

\section{Acknowledgements}

B.P. and Y.-L.H. contributed equally to this work. B.P. was supported by an Intel grant; Y.-L.H. was supported by a grant from the U.S. Department of Energy Advanced Manufacturing Office. Z.C. acknowledges partial support of the National Science Foundation under Grant DMR-1708615 and the Laboratory Directed Research and Development Program of Lawrence Berkeley National Laboratory under U.S. Department of Energy (DOE) Contract No. DE-AC02-05CH11231. B.D.H. and J.S. acknowledge an NSF: DMR: MRI Development grant 1726862. J.I. is supported by the Luxembourg National Research Fund through the CORE program (Grant FNR/C18/MS/12705883 REFOX). L.W.M. and R.R. acknowledge the support of Intel Corp. through the FEINMAN program. Q.L., M.Y., and Z.Q.Q. acknowledge support by US Department of Energy, Office of Science, Office of Basic Energy Sciences, Materials Sciences and Engineering Division under Contract No. DE-AC02-05CH11231 (van der Waals heterostructures program, KCWF16). This research used resources of the Advanced Light Source, which is a DOE Office of Science User Facility under contract no. DE-AC02-05CH11231.

\section{Conflict of Interest}

The authors declare no conflict of interest.

\section{Keywords}

magnetoelectrics, multiferroics, nonvolatile memories, spintronics, ultralow-power spintronics

Received: March 20, 2020

Revised: April 27, 2020

Published online:

[1] A. V Chumak, V. I. Vasyuchka, A. A. Serga, B. Hillebrands, Nat. Phys. 2015, 11, 453.

[2] M. Bibes, A. Barthélémy, Nat. Mater. 2008, 7, 425.

[3] N. A. Spaldin, R. Ramesh, Nat. Mater. 2019, 18, 203.

[4] J. D. Meindl, Q. Chen, J. A. Davis, Science 2001, 293, 2044.

[5] T. N. Theis, P. M. Solomon, Science 2010, 327, 1600.

[6] I. Ferain, C. A. Colinge, J. P. Colinge, Nature 2011, 479, 310

[7] H. Li, B. Gao, Z. Chen, Y. Zhao, P. Huang, H. Ye, L. Liu, X. Liu, J. Kang, Sci. Rep. 2015, 5, 13330.

[8] G. Sun, J. Zhao, M. Poremba, C. Xu, Y. Xie, Natl. Sci. Rev. 2018, 5, 577.

[9] S. Manipatruni, D. E. Nikonov, I. A. Young, Nat. Phys. 2018, 14, 338.

[10] O. Golonzka, J. Alzate, U. Arslan, M. Bohr, P. Bai, J. Brockman, B. Buford, C. Connor, N. Das, B. Doyle, T. Ghani, F. Hamzaoglu, 
P. Heil, P. Hentges, R. Jahan, D. Kencke, B. Lin, M. Lu, M. Mainuddin, M. Meterelliyoz, P. Nguyen, D. Nikonov, K. O'brien, J. ODonnell, K. Oguz, D. Ouellette, J. Park, J. Pellegren, C. Puls, P. Quintero, T. Rahman, A. Romang, M. Sekhar, A. Selarka, M. Seth, A. J. Smith, A. K. Smith, L. Wei, C. Wiegand, Z. Zhang, K. Fischer, in IEEE Int. Electron Devices Meeting, IEEE, Piscataway, N), USA 2018, pp. 18.1.1-18.1.4.

[11] H. S. P. Wong, S. Salahuddin, Nat. Nanotechnol. 2015, 10, 191.

[12] S. Manipatruni, D. E. Nikonov, C.-C. Lin, T. A. Gosavi, H. Liu, B. Prasad, Y.-L. Huang, E. Bonturim, R. Ramesh, I. A. Young, Nature 2019, 565, 35.

[13] C. Ederer, N. A. Spaldin, Phys. Rev. B 2005, 71, 06040

[14] B. K. Jang, J. H. Lee, K. Chu, P. Sharma, G. Y. Kim, K. T. Ko, K. E. Kim, Y. J. Kim, K. Kang, H. B. Jang, H. Jang, M. H. Jung, K. Song, T. Y. Koo, S. Y. Choi, J. Seidel, Y. H. Jeong, H. Ohldag, J. S. Lee, C. H. Yang, Nat. Phys. 2017, 13, 189.

[15] S. V. Kalinin, Nat. Phys. 2017, 13, 115.

[16] O. E. González-Vázquez, J. C. Wojdeł, O. Diéguez, J. Añiguez, Phys. Rev. B 2012, 85, 064119.

[17] Y. H. Chu, Q. Zhan, C. H. Yang, M. P. Cruz, L. W. Martin, T. Zhao, P. Yu, R. Ramesh, P. T. Joseph, I. N. Lin, W. Tian, D. G. Schlom, Appl. Phys. Lett. 2008, 92, 90.
[18] T. Zhao, A Scholl, F. Zavaliche, K. Lee, M. Barry, A Doran, M. P. Cruz, Y. H. Chu, C. Ederer, N. A. Spaldin, R. R. Das, D. M. Kim, S. H. Baek, C. B. Eom, R. Ramesh, Nat. Mater. 2006, 5, 823.

[19] J. J. Steffes, R. A. Ristau, R. Ramesh, B. D. Huey, Proc. Natl. Acad. Sci. USA 2019.

[20] S. Yu, P. Y. Chen, IEEE Solid-State Circuits Mag. 2016, 8, 43.

[21] K. L. Wang, J. G. Alzate, P. K. Amiri, J. Phys. D: Appl. Phys. 2013, 46, 074003.

[22] H. Cai, W. Kang, Y. Wang, N. Lirida, J. Yang, Z. Weisheng, Appl. Sci. 2017, 7, 929.

[23] J. Junquera, P. Ghosez, Nature 2003, 422, 506.

[24] D. D. Fong, G. B. Stephenson, S. K. Streiffer, J. A. Eastman, O. Auciello, P. H. Fuoss, C. Thompson, Science 2004, 304, 1650.

[25] P. Maksymovych, M. Huijben, M. Pan, S. Jesse, N. Balke, Y. H. Chu, H. J. Chang, A. Y. Borisevich, A. P. Baddorf, G. Rijnders, D. H. A. Blank, R. Ramesh, S. V. Kalinin, Phys. Rev. B 2012, 85, 014119.

[26] G. Kresse, J. Furthmüller, Phys. Rev. B 1996, 54, 11169.

[27] J. P. Perdew, K. Burke, M. Ernzerhof, Phys. Rev. Lett. 1996, 77, 3865.

[28] O. Diéguez, O. E. González-Vázquez, J. C. Wojdeł, J. Íñiguez, Phys. Rev. B 2011, 83, 094105

[29] S. L. Dudarev, G. A. Botton, S. Y. Savrasov, C. J. Humphreys, A. P. Sutton, Phys. Rev. B 1998, 57, 1505.

[30] P. E. Blöchl, Phys. Rev. B 1994, 50, 17953. 